\title{
Prediction of Electrical Conductivity of Deep Eutectic Solvents using COSMO-RS Sigma Profiles as Molecular Descriptors: A Quantitative Structure-Property Relationship Study
}

\section{Supporting Information}

Tarek Lemaoui ${ }^{\mathrm{a}, 1}$, Ahmad S. Darwish ${ }^{\mathrm{b}, 1}$, Nour El Houda Hammoudia ${ }^{\mathrm{a}}$, Farah Abu Hatab ${ }^{\mathrm{b}}$, Ayoub Attoui ${ }^{\mathrm{a}}$, Inas M. Alnashef ${ }^{b}$, Yacine Benguerba ${ }^{a}$ *

aLaboratoire des Matériaux Polymères Multiphasiques (LMPMP), Université Ferhat ABBAS Sétif-1, 19000

$$
\text { Sétif, Algeria }
$$

${ }^{\mathrm{b}}$ Khalifa University of Science and Technology, Center for Membrane and Advanced Water Technology (CMAT), Abu Dhabi, P.O. Box 127788, United Arab Emirates (UAE)

${ }^{1}$ The authors have equally contributed to this work

*Corresponding Author: Yacine Benguerba, yacinebenguerba@univ-setif.dz, T: +213-561535093 
Table S.1: Experimental electrical conductivity data $\left[\mathrm{mS} \cdot \mathrm{cm}^{-1}\right]$ of the DESs used in this work measured at $\mathrm{P}=1.01$ bar. Data were taken from references ${ }^{\mathrm{S} 1, \mathrm{~S} 2}$.

\begin{tabular}{|c|c|c|c|}
\hline $\mathrm{T}(\mathrm{K})$ & $\mathrm{k}\left(\mathrm{mS} . \mathrm{cm}^{-1}\right)$ & $\mathrm{T}(\mathrm{K})$ & $\mathrm{k}\left(\mathrm{mS} \cdot \mathrm{cm}^{-1}\right)$ \\
\hline \multicolumn{2}{|c|}{ DES 1, BTPPCl:EG (1:3) } & \multicolumn{2}{|c|}{ DES 2, BTPPCl:Gly (1:5) } \\
\hline 328.15 & 0.485 & 328.15 & 0.1628 \\
\hline 338.15 & 0.1989 & 338.15 & 0.0187 \\
\hline 348.15 & 0.0164 & 348.15 & 0.0167 \\
\hline 358.15 & 0.0148 & 358.15 & 0.0151 \\
\hline 368.15 & 0.0136 & 368.15 & 0.0139 \\
\hline \multicolumn{2}{|c|}{ DES 3, MTPPBr:EG (1:5.25) } & \multicolumn{2}{|c|}{ DES 4, MTPPBr:EG (1:4) } \\
\hline 298.15 & 1.942 & 298.15 & 1.557 \\
\hline 303.15 & 2.57 & 303.15 & 2.193 \\
\hline 308.15 & 3.103 & 308.15 & 2.649 \\
\hline 313.15 & 3.845 & 313.15 & 3.246 \\
\hline 318.15 & 4.437 & 318.15 & 3.858 \\
\hline 323.15 & 5.072 & 323.15 & 4.405 \\
\hline 328.15 & 6.279 & 328.15 & 5.395 \\
\hline 333.15 & 7.11 & 333.15 & 6.221 \\
\hline 338.15 & 8.169 & 338.15 & 7.423 \\
\hline 343.15 & 9.496 & 343.15 & 8.192 \\
\hline 348.15 & 10.31 & 348.15 & 9.074 \\
\hline 353.15 & 11.196 & 353.15 & 10.027 \\
\hline \multicolumn{2}{|c|}{ DES 5, MTPPBr:EG (1:3) } & \multicolumn{2}{|c|}{ DES 6, MTPPBr:Gly (1:4) } \\
\hline 298.15 & 1.092 & 298.15 & 0.116 \\
\hline 303.15 & 1.598 & 303.15 & 0.198 \\
\hline 308.15 & 1.9136 & 308.15 & 0.37 \\
\hline 313.15 & 2.502 & 313.15 & 0.41 \\
\hline 318.15 & 2.964 & 318.15 & 0.607 \\
\hline 323.15 & 3.265 & 323.15 & 0.816 \\
\hline 328.15 & 4.307 & 328.15 & 0.965 \\
\hline 333.15 & 5.129 & 333.15 & 1.233 \\
\hline 338.15 & 5.797 & 338.15 & 1.608 \\
\hline 343.15 & 6.723 & 343.15 & 1.971 \\
\hline 348.15 & 7.372 & 348.15 & 2.874 \\
\hline 353.15 & 8.114 & 353.15 & 3.594 \\
\hline
\end{tabular}




\begin{tabular}{|c|c|c|c|}
\hline \multicolumn{2}{|c|}{ DES 7, MTPPBr:Gly (1:3) } & \multicolumn{2}{|c|}{ DES 8, MTPPBr:Gly (1:1.75) } \\
\hline 298.15 & 0.103 & 298.15 & 0.062 \\
\hline 303.15 & 0.172 & 303.15 & 0.124 \\
\hline 308.15 & 0.319 & 308.15 & 0.186 \\
\hline 313.15 & 0.394 & 313.15 & 0.277 \\
\hline 318.15 & 0.549 & 318.15 & 0.405 \\
\hline 323.15 & 0.719 & 323.15 & 0.496 \\
\hline 328.15 & 0.927 & 328.15 & 0.701 \\
\hline 333.15 & 1.124 & 333.15 & 0.858 \\
\hline 338.15 & 1.487 & 338.15 & 1.16 \\
\hline 343.15 & 1.778 & 343.15 & 1.493 \\
\hline 348.15 & 2.196 & 348.15 & 1.811 \\
\hline 353.15 & 2.599 & 353.15 & 2.154 \\
\hline \multicolumn{2}{|c|}{ DES 9, MTPPBr:TFA (1:8) } & \multicolumn{2}{|c|}{ DES 10, ChCl:EG (1:2.5) } \\
\hline & & 298.15 & 8.317 \\
\hline 278.15 & 0.1343 & 303.15 & 10.665 \\
\hline 288.15 & 0.351 & 308.15 & 12.07 \\
\hline 298.15 & 0.848 & 313.15 & 14.133 \\
\hline 308.15 & 1.821 & 318.15 & 16.558 \\
\hline 318.15 & 2.82 & 323.15 & 17.977 \\
\hline 328.15 & 3.49 & 328.15 & 21.257 \\
\hline 338.15 & 4.18 & 333.15 & 24.247 \\
\hline 348.15 & 4.82 & 338.15 & 25.152 \\
\hline 358.15 & 5.5 & 343.15 & 26.275 \\
\hline \multirow[t]{2}{*}{368.15} & 6.09 & 348.15 & 27.799 \\
\hline & & 353.15 & 28.69 \\
\hline \multicolumn{2}{|c|}{ DES 11, ChCl:EG (1:2) } & \multicolumn{2}{|c|}{ DES 12, ChCl:EG (1:1.75) } \\
\hline 298.15 & 7.332 & 298.15 & 6.801 \\
\hline 303.15 & 10.191 & 303.15 & 9.138 \\
\hline 308.15 & 11.407 & 308.15 & 10.857 \\
\hline 313.15 & 13.553 & 313.15 & 12.935 \\
\hline 318.15 & 15.895 & 318.15 & 14.794 \\
\hline 323.15 & 17.185 & 323.15 & 16.335 \\
\hline 328.15 & 20.227 & 328.15 & 18.393 \\
\hline 333.15 & 22.991 & 333.15 & 20.102 \\
\hline 338.15 & 24.2 & 338.15 & 21.773 \\
\hline 343.15 & 25.599 & 343.15 & 23.474 \\
\hline 348.15 & 27.065 & 348.15 & 24.75 \\
\hline 353.15 & 28.072 & 353.15 & 26.043 \\
\hline
\end{tabular}




\begin{tabular}{|c|c|c|c|}
\hline \multicolumn{2}{|c|}{ DES 13, ChCl:Gly (1:3) } & \multicolumn{2}{|c|}{ DES 14, ChCl:Gly (1:2) } \\
\hline 298.15 & 1.463 & 298.15 & 1.749 \\
\hline 303.15 & 1.553 & 303.15 & 1.951 \\
\hline 308.15 & 2.035 & 308.15 & 2.549 \\
\hline 313.15 & 2.57 & 313.15 & 3.004 \\
\hline 318.15 & 3.112 & 318.15 & 3.991 \\
\hline 323.15 & 3.816 & 323.15 & 5.12 \\
\hline 328.15 & 4.811 & 328.15 & 6.046 \\
\hline 333.15 & 5.757 & 333.15 & 7.187 \\
\hline 338.15 & 6.717 & 338.15 & 8.16 \\
\hline 343.15 & 7.805 & 343.15 & 8.955 \\
\hline 348.15 & 9.286 & 348.15 & 10.629 \\
\hline 353.15 & 10.8 & 353.15 & 12.191 \\
\hline \multicolumn{2}{|c|}{ DES 15, ChCl:Gly (1:1) } & \multicolumn{2}{|c|}{ DES 16, DEACl:EG (1:4) } \\
\hline 298.15 & 1.929 & 298.15 & 5.661 \\
\hline 303.15 & 2.191 & 303.15 & 6.994 \\
\hline 308.15 & 3.161 & 308.15 & 8.245 \\
\hline 313.15 & 4.603 & 313.15 & 9.699 \\
\hline 318.15 & 5.864 & 318.15 & 11.579 \\
\hline 323.15 & 6.668 & 323.15 & 13.408 \\
\hline 328.15 & 7.805 & 328.15 & 15.086 \\
\hline 333.15 & 8.98 & 333.15 & 17.137 \\
\hline 338.15 & 9.863 & 338.15 & 18.755 \\
\hline 343.15 & 11.548 & 343.15 & 20.286 \\
\hline 348.15 & 11.548 & 348.15 & 22.262 \\
\hline 353.15 & 12.954 & 353.15 & 24.053 \\
\hline \multicolumn{2}{|c|}{ DES 17, DEACl:EG (1:3) } & \multicolumn{2}{|c|}{ DES 18, DEACl:EG (1:2.5) } \\
\hline 298.15 & 5.429 & 298.15 & 5.12 \\
\hline 303.15 & 6.878 & 303.15 & 6.627 \\
\hline 308.15 & 8.305 & 308.15 & 7.94 \\
\hline 313.15 & 9.486 & 313.15 & 9.283 \\
\hline 318.15 & 11.339 & 318.15 & 10.955 \\
\hline 323.15 & 13.147 & 323.15 & 12.539 \\
\hline 328.15 & 14.769 & 328.15 & 14.33 \\
\hline 333.15 & 16.664 & 333.15 & 16.161 \\
\hline 338.15 & 18.395 & 338.15 & 17.779 \\
\hline 343.15 & 19.9 & 343.15 & 19.417 \\
\hline 348.15 & 21.924 & 348.15 & 21.347 \\
\hline 353.15 & 23.667 & 353.15 & 23.097 \\
\hline
\end{tabular}




\begin{tabular}{lccc}
\hline \multicolumn{2}{c}{ DES 19, DEACl:Gly (1:4) } & \multicolumn{2}{c}{ DES 20, DEACl:Gly (1:3) } \\
\hline 298.15 & 0.487 & 298.15 & 0.602 \\
303.15 & 0.78 & 303.15 & 0.958 \\
308.15 & 1.099 & 308.15 & 1.041 \\
313.15 & 1.387 & 313.15 & 1.562 \\
318.15 & 1.878 & 318.15 & 2.112 \\
323.15 & 2.357 & 323.15 & 2.637 \\
328.15 & 2.716 & 328.15 & 3.426 \\
333.15 & 3.246 & 333.15 & 4.086 \\
338.15 & 3.962 & 338.15 & 4.916 \\
343.15 & 4.646 & 343.15 & 5.748 \\
348.15 & 5.335 & 348.15 & 6.474 \\
353.15 & 6.095 & 353.15 & 7.1
\end{tabular}

\begin{tabular}{cc}
\hline \multicolumn{2}{c}{ DES 21, DEACl:Gly (1:2) } \\
\hline 298.15 & 0.75 \\
303.15 & 1.177 \\
308.15 & 1.635 \\
313.15 & 2.067 \\
318.15 & 2.716 \\
323.15 & 3.381 \\
328.15 & 3.903 \\
333.15 & 4.878 \\
338.15 & 5.754 \\
343.15 & 6.521 \\
348.15 & 7.754 \\
353.15 & 9.109 \\
\hline
\end{tabular}

\section{Abbreviations}

$\begin{array}{ll}\text { BTPPCl } & \text { Benzyltriphenylphosphonium Chloride } \\ \text { ChCl } & \text { Choline Chloride } \\ \text { DEACl } & \text { N,N-diethyl ethanol ammonium Chloride } \\ \text { MTPPBr } & \text { Methyltriphenylphosphonium Bromide } \\ \text { EG } & \text { Ethylene Glycol } \\ \text { Gly } & \text { Glycerol } \\ \text { TFA } & 2,2,2 \text {-Trifluoroacetamide }\end{array}$




\section{References}

(S1) Bagh, F. S. G.; Shahbaz, K.; Mjalli, F. S.; AlNashef, I. M.; Hashim, M. A. Electrical Conductivity of Ammonium and Phosphonium Based Deep Eutectic Solvents: Measurements and Artificial Intelligence-Based Prediction. Fluid Phase Equilib. 2013, 356, 30-37. https://doi.org/10.1016/j.fluid.2013.07.012.

(S2) Kareem, M. A.; Mjalli, F. S.; Hashim, M. A.; Alnashef, I. M. Phosphonium-Based Ionic Liquids Analogues and Their Physical Properties. J. Chem. Eng. Data 2010. https://doi.org/10.1021/je100104v. 